\title{
Plasticidad fenotípica en mandíbulas de Heliconius erato (Lepidoptera: Nymphalidae) inducida por diferentes plantas hospederas (Passifloraceae)
}

\author{
Phenotypic plasticity in Heliconius erato (Lepidoptera: Nymphalidae) mandibles induced \\ by different host plants (Passifloraceae)
}

CAROLINA MILLÁN ${ }^{1}$, RODRIGO FORNEL ${ }^{2}$ y GILSON R. P. MOREIRA ${ }^{3}$

\begin{abstract}
Resumen: El objetivo fue analizar si el consumo de diferentes plantas influenciaba el tamaño/forma de las mandíbulas de larvas de lepidópteros oligófagas y si dicho consumo ocasionaba tasas de crecimiento diferenciadas entre el músculo aductor mandibular y las mandíbulas durante su ontogénesis. Para esto se usaron larvas de Heliconius erato criadas en cuatro plantas hospederas naturales, a las que se les tomaron medidas de longitud de cápsula cefálica, tamaño mandibular y área del músculo aductor. Para detectar eventuales diferencias de forma mandibular se empleó microscopía electrónica y morfometria geométrica, y para posibles cambios alométricos se realizaron comparaciones entre curvas de crecimiento mandíbula vs. músculo aductor de los individuos criados en las cuatro plantas hospederas. Se encontró que no existen diferencias de tamaño entre las mandíbulas ni en músculos aductores de individuos criados en las diferentes plantas pero la forma mandibular fue significativamente diferente, mostrando una relación directa con la planta hospedera usada como alimento. Generalmente, la mandíbula creció proporcionalmente más que el músculo aductor, cuyo crecimiento fue estable. El cambio más significativo fue el alargamiento de los dientes incisivos en mandíbulas de larvas criadas con Passiflora suberosa. Se sugiere que esto se debe a que las hojas de esta especie poseen mayor dureza comparadas con las otras y que el desarrollar dientes largos facilitaría su corte y consumo.
\end{abstract}

Palabras clave: Mariposas heliconíneos, morfologia larval, alometria mandibular, adaptaciones alimenticias.

\begin{abstract}
This study aimed to test whether the consumption of different host plants influences the size and shape of mandibles and associated muscles in oligophagic insects throughout ontogeny. Larvae of Heliconius erato phyllis (Lepidoptera: Nymphalidae) were reared on four species of passion vines (Passifloraceae) and had the size of their head caspules, mandibles and associated adductor muscles measured afterward. Corresponding effects on shape and potential allometries in mandibles were also evaluted using geometric morphometric analysis based on scanning electron micrographs. Growth in mandibular size was greater compared to that of associated adductor muscles. There was no host plant effect on the sizes of either mandibles or adductor muscles. However, the shapes of mandibles was significantly different among passion vine species used as larval food. Greater differences in shape associated with wider teeth were obtained for Passiflora suberosa, whose leaves are tougher compared to those of $P$. misera, $P$. caerulea, and P. actinia; and, among which there was no difference in manbilular shape. Thus, it was inferred that the larval stage of this Heliconius species is able to adjust its mandible shape according to the toughness of host plants; a phenotypic plasticity mechanism that can be supposed to have evolved in order to facilitate processing these kinds of leaves.
\end{abstract}

Key words: Heliconian butterflies, larvae morphology, mandibular allometry, feeding adaptations.

\section{Introducción}

En un abordaje ecológico, de plasticidad fenotípica en animales se define como un grupo de expresiones fenotípicas dependientes del ambiente que obedecen a un ajuste del individuo a su entorno (Bradshaw 1965; Schilichting 1986; Sultan 1995; Pigliucci 2002; West-Eberhard 2003; DeWitt y Scheiner 2004). Representa un poderoso medio de adaptación en el cual alelos alternativos (o sus productos) reaccionan de manera diferente al medio ambiente; aquellos con reacciones favorables persisten y los otros desaparecen. Frecuente y tradicionalmente la plasticidad fenotípica se interpreta como una expresión "no genética" (Wright 1931). Las dudas sobre sí las variaciones fenotípicas de los organismos en determi- nados ambientes son plásticas o genéticas son persistentes y engañosas debido a esta visión incorrecta de que efectos ambientales y genéticos son independientes. Los genes y el ambiente pueden interactuar y las respuestas fenotípicas son genéticas en todos los casos; la diferencia radica en sí las mutaciones que surgieron son favorecidas (heredadas) o no (DeWitt y Scheiner 2004).

Desde décadas, el papel de la plasticidad fenotípica en la adaptación a ambientes inciertos ha sido foco de varios estudios. Sin embargo, a pesar del volumen grande de artículos en este campo, la plasticidad adaptativa no ha sido ampliamente documentada (Scheiner 1993; Gotthard y Nylin 1995). Las interacciones entre insectos y sus hospederos vegetales conforman un sistema complejo y altamente dinámico (Ber-

\footnotetext{
${ }^{1}$ Estudiante Ph. D. Departamento de Ecologia, Instituto de Biociências, Universidade Federal do Rio Grande do Sul. Avenida Bento Gonçalves 9500, 91501-970 Porto Alegre, Rio Grande do Sul, Brasil, lepidoptera.azul@gmail.com. ${ }^{2}$ Profesor, Ph. D. Departamento de Ciências Biológicas, Laboratório de Ecomorfología Animal, Universidade Regional Integrada do Alto Uruguai e das Missões (URI), Rua Sete de Setembro, 1621, 99709-910, Erechim, Brasil, rodrigofornel@hotmail.com. ${ }^{3}$ Profesor, Ph. D. Departamento de Zoologia, Instituto de Biociências, Universidade Federal do Rio Grande do Sul. Avenida Bento Gonçalves 9500, 91501-970 Porto Alegre, Rio Grande do Sul, Brasil, gilson.moreira@ufrgs.br. Autor para correspondencia: Carolina Millan. Estudiante Ph. D. Departamento de Ecologia, Instituto de Biociências, Universidade Federal do Rio Grande do Sul. Avenida Bento Gonçalves 9500, $91501-970$ Porto Alegre, Rio Grande do Sul, Brasil, lepidoptera.azul@gmail.com.
} 
nays 1998) que puede inducir modificaciones fenotípicas a corto plazo que afectan el desarrollo y la evolución de sus estructuras corporales, inclusive sus piezas bucales (MennaBarreto y Araújo 1985; Périco y Araújo 1991; Price 1997; Bernays 1998; Larsson 2002). Bernays (1986) usando larvas de Spodoptera (Lepidoptera: Noctuidae) y algunas especies de grillos, demostró que las dimensiones de la cápsula cefálica y músculos mandibulares están intimamente relacionados con el tipo de dieta. En este sentido, la autora también mostró alometría en larvas de Pseudaletia unipuncta (Haworth, 1809) (Lepidoptera: Noctuidae) sometidas a diferentes dietas, donde la alimentación con tejido rígido provocó un aumento proporcional mayor de la cabeza con relación al tamaño del cuerpo.

Las mandíbulas de insectos masticadores juegan un papel decisivo en su desarrollo al establecer el primer contacto con el tejido vegetal y al encargarse de cortarlo en fragmentos, determinando si puede o no ser ingerido. En ese sentido, las mandíbulas pueden sufrir presiones selectivas importantes (Chapman 1998). Generalmente, las mandíbulas presentan dos áreas distintas en su morfología externa: una parte distal o 'región incisiva' que contiene los dientes incisivos y es responsable por cortar el alimento; y una área proximal o 'región molar' que lo tritura (Chapman 1995). Internamente, la anatomía de las mandíbulas ha sido estudiada en ortópteros (Baines et al. 1990), himenópteros (Masuko 1986; Rehder 1989; Paul y Gronenberg 2002) y larvas de Sphingidae (Griss 1990). En lepidópteros que poseen mandíbulas dicóndilas y aparatos bucales hipognatos, existe un par de músculos insertados en apodemas que están localizados a cada lado del plano de articulación y se encargan de los movimientos de abducción y aducción (Snodgrass 1935; 1950). Dichos músculos se originan en la porción antero-dorsal de la cabeza, donde el músculo abductor se encarga de la rapidez de contracción mientras el aductor proporciona la fuerza de cierre a las mandíbulas, siendo responsable de generar gran parte de la fuerza empleada para cortar el alimento. Debido a eso, el aductor es más grande que el abductor y ocupa, prácticamente, toda la cavidad cefálica (Snodgrass 1935; Chapman 1995; Vegliante 2005).

Las mariposas de la tribu Heliconiini (Lepidoptera: Nymphalidae) han sido utilizadas en diversos estudios evolutivos y son reconocidas principalmente por la estrecha relación con sus plantas hospederas (Passifloraceae). Durante mucho tiempo este complejo fue propuesto como un sistema de evolución conjunta (Gilbert 1975; Benson et al. 1976; Benson 1978).

Alexander (1961) describió diferentes estrategias adoptadas por larvas de seis especies de heliconíneos que rompen la nervadura central de las hojas y raspan tejidos superficiales de las mismas para poder alimentarse, sugiriendo que dichas estrategias estarían relacionadas no solamente con el comportamiento de los individuos sino también con la forma de sus mandíbulas. Décadas después, Aymone (2009) registró cambios de forma convergentes en la cabeza de ocho especies de heliconíneos durante su trayectoria ontogenética, lo que relacionó con el crecimiento gradual de las mandíbulas y músculos aductores en la cabeza de estas mariposas. Al igual que Alexander (1961), Aymone sugirió que dicha convergencia estaría relacionada a los hábitos alimenticios (convergentes) adoptados por las especies durante su trayectoria evolutiva; dichos hábitos fueron descritos detalladamente por Benson (1978). Sin embargo, aunque se reconoce que la variación en el uso de la planta hospedera está asociada a plasticidad fenotípica en estos insectos, por lo menos en relación al tamaño y forma de las alas (Jorge et al. 2011), se desconoce la existencia de tal relación a los respectivos apéndices alimenticios.

Heliconius erato phyllis (Fabricus, 1775) es una especie oligófaga cuyos individuos tienen una alta orientación espacial con relación a las plantas hospederas y una acentuada capacidad de inspeccionar su calidad (Mugrabi-Oliveira y Moreira 1996 a, b). Las hembras adultas ponen huevos exclusivamente en hojas jóvenes, que son más suaves, y en los zarcillos (Mugrabi-Oliveira y Moreira 1996 a, b), más blandos que las hojas maduras, que se encuentran en la base del ramo (Fenny 1970; Barp et al. 2006; Elpino-Campos 2010). En estudios comportamentales Rodrigues y Moreira (1999) observaron que, en condiciones naturales, larvas del primer y segundo instar se alimentan de tejidos jóvenes (suaves) localizados en la parte superior del ramo de Passiflora y, a medida que ocurre la ontogénesis, las larvas avanzan hacia la parte posterior del ramo donde se encuentran las hojas más rígidas. Los autores también registraron una mortalidad significativa de las larvas jóvenes cuando fueron obligadas a alimentarse de hojas maduras. Posteriormente, Silveira (2002) y ElpinoCampos (2010) atribuyeron dicha mortalidad acentuada a que las mandíbulas de los individuos jóvenes serian incapaces de masticar hojas duras.

Interrogantes relacionados con la descripción y cuantificación de procesos evolutivos que implican variaciones en las tasas de crecimento y posibles cambios de forma que sufrirían las mandíbulas de los heliconíneos frente al consumo de diferentes pasifloras permanecen inexploradas. Los objetivos de este trabajo fueron 1) determinar si el consumo de diferentes plantas por parte de larvas de $H$. erato influenciaba el tamaño/forma mandibulares y las dimensiones del músculo aductor asociado a las mismas y 2) determinar si existían diferencias en las tasas de crecimiento entre mandíbulas y músculos aductores mandibulares que puedieran ser atribuidas al consumo de las diferentes plantas. Las hipótesis de trabajo fueron que 1) las larvas de $H$. erato presentarían diferencias de tamaño y forma de las mandíbulas y también diferencias en el área del músculo aductor como una respuesta fenotípica al consumo de diferentes plantas y que por ese motivo, 2) las larvas de $H$. erato presentarían diferentes tasas de crecimiento de mandibula y el músculo aductor.

\section{Materiales y métodos}

Cría de larvas y cultivo de pasifloráceas. Todas las larvas de $H$. erato utilizadas en este trabajo se obtuvieron a partir de huevos de hembras acondicionadas en insectarios y que fueron recolectadas en diferentes localidades de Porto Alegre, Brasil. Las larvas se mantuvieron en condiciones ambientales controladas en el Departamento de Zoología de la Universidad Federal do Rio Grande do Sul (UFRGS), alimentadas $a d$ libitum en ramos intactos de cuatro especies de Passiflora.

En la región de Rio Grande do Sul (Brasil) se registran nueve especies de Passiflora como hospederas de H. erato: Passiflora actinia Hook, P. alata Curtis, P. caerulea Linnaeus, $P$. capsularis Linnaeus, $P$. edulis Sims, $P$. elegans Mast, $P$. misera Kunt, $P$. suberosa Linnaeus y $P$. tenuifila Killip (Sacco 1962, 1980). Dentro de esas plantas, P. edulis junto con $P$. alata y $P$. tenuifila son consideradas hospederas letales debido a las defensas químicas que presentan (Périco y Araújo 1991). Con base en la información anterior se usa- 
ron en el experimento las especies $P$. actinia, $P$. caerulea, $P$. misera y $P$. suberosa, las cuales se obtuvieron de un cultivo de pasifloras establecido especialmente para dicho fin en la misma localidad de estudio.

El material montado y analizado en este estudio reposa en la colección del Laboratorio de Morfologia y Comportamiento de Insectos (LMCI) de la Universidad Federal do Rio Grande do Sul, Porto Alegre, bajo el código de acceso LMCI 250.

Preparación de material en laboratorio. Para hacer el seguimiento de la forma mandibular de $H$. erato en el transcurso de su ontogénesis y poder asignar las coordenadas para los análisis de este estudio, se criaron larvas de todos los instares en Passiflora suberosa ( $\mathrm{n}=12$ /instar) que es la planta más utilizada por esta mariposa en condiciones naturales. Dos horas después de haber alcanzado cada instar larval (L1 - L5) los invidividuos fueron congelados y se disectaron todas las cabezas, usando un corte sagital central. Para descartar posibles asimetrías y estandarizar las comparaciones morfológicas entre las mandíbulas, se utilizó solamente la mitad izquierda de la cabeza. Individualmente, cada mitad se colocó en posición horizontal, encima de láminas de microscopía forradas con cinta doble faz; se realizaron fotografías en vista frontal con una cámara digital Nikon ${ }^{\circledR}$ DS-U3 adaptada a un microscopio Nikon ${ }^{\circledR}$ AZ100M, utilizando el software de imagen NISElements y en este mismo software se realizaron medidas de longitud cefálica $(\mathrm{mm})$, considerada desde la inserción del condilo anterior hasta la base del escolus.

Para probar si el consumo de diferentes plantas influenciaba el tamaño y la forma de las mandíbulas, se emplearon larvas adicionales de $H$. erato alimentadas hasta quinto instar $($ L5) en $P$. actinia $(\mathrm{n}=12), P$. caerulea $(\mathrm{n}=14), P$, misera $(\mathrm{n}$ $=16)$ y $P$. suberosa $(\mathrm{n}=14)$, siguiendo el mismo proceso de disección y montaje descritos. Se utilizaron solamente larvas de quinto instar ya que, independiente de que las mandíbulas son sustituidas a través de las mudas, posibles consecuencias alométricas y morfológicas ocasionadas por la planta hospedera serian evidentes solamente al final de la ontogénesis.

Para analizar el músculo aductor mandibular se usaron solamente las mitades derechas de las cabezas de los individuos sometidos a las diferentes dietas y posteriormente dichas mitades se prepararon siguiendo el mismo procedimiento de montaje descrito arriba. Con el software NIS-Elements se calculó el área de los músculos aductores usando medidas de $\mathrm{R}$ (diámetro del músculo aductor), Hf (altura frontal del músculo aductor) y Hd (altura dorsal del músculo aductor) según lo realizado por Aymone (2006). Para este experimento también se utilizaron solamente individuos de quinto instar pues el músculo aductor asociado a las mandíbulas no se pierde ni es reemplazado durante las mudas y, por lo tanto, las posibles consecuencias de cualquier tratamiento proporcionado a las larvas sería percibido con mayor fuerza al final de la ontegenesis.

Analisis morfométricos. Una vez se tomaron las fotos y las medidas a todos los individuos, se realizaron comparaciones entre las longitudes cefálicas, tamaño de las mandíbulas y áreas de los músculos aductores. No se cumplieron los supuestos de normalidad (Tests Shapiro-Wilk) y homegenidad de varianza (Test de Hartley), por lo que dichas comparaciones se analizaron con estadística no paramétrica. Las alometrías en las mandíbulas y músculos aductores de las larvas de quinto instar se analizaron usando corelación de Pearson y comparaciones ANCOVA (GraphPadPrism 5.00) entre las rectas de crecimiento correpondientes.

Para el análisis de la forma mandibular, la digitación de los "landmarks" y su localización en coordenadas cartesianas se hizo sobre las fotos obtenidas en laboratorio, utilizando el programa TPSdig 2.16 (Rohlf 2010); siendo asignados 17 coordenadas consideradas regiones anatómicas de interés (Tabla 1) (Fig. 1). Dichas coordenadas se analizaron con el método de sobreposición de Procustes (Rohlf y Slice 1990) y análisis de componentes principales (PCA) seguidos de análisis discriminantes (CVA). La significancia de variación ontogenética se calculó por medio de análisis multivariados MANOVA y los análisis de distribucion de las formas mandibulares en los instares y en las plantas se hicieron mediante PCA y posterior MANOVA.

Además, se realizó un análisis de deformaciones relativas (Rohlf 2006) gráficamente a través de la función "Thin Plate Splines" (Adams y Rosenberg 1998) y se establecieron relaciones de proximidad fenotípica a través de distancias de Mahalanobis (1936) y árboles del vecino cercano "neighborjoining" (Saitou y Nei 1987) no enraizadas. Los análisis se rodaron en el programa " $R$ ", usando Rmorph package (Baylac 2008) y los paquetes MASS, Ape, ade4 y stats (Development Core Team 2008).

\section{Resultados}

Las mandíbulas de larvas de $H$. erato fueron de tipo cortador/ masticador (Alexander 1961) durante toda su ontogénesis (Fig. 1) y los índices de identificación correcta para la deter-

Tabla 1. Descripción de las regiones mandibulares y respectivos puntos anatómicos utilizados en los análisis morfométricos.

\begin{tabular}{lcl}
\hline Región & Coordenada "Landmark” & \multicolumn{1}{c}{ Descripción } \\
\hline Condilar & 1 & Cóndilo posterior de la mandíbula. \\
& 2 & Vértice de la margen distal, localizado entre los dos cóndilos mandibulares. \\
& 3 & Cóndilo anterior de la mandíbula. \\
Molar & 5 & Límite lateral izquierdo de la margen establecida por la región molar. \\
& 6 & Límite proximal del diente 11 - 12 con la margen de la región molar. \\
Incisiva & $7,9,11,13,15$ y 17 & Límite proximal del diente 9 - 10 com a margem de la región molar. \\
& $8,10,12,14$ y 16 & Base externa entre los dientes incisivos. \\
\hline
\end{tabular}



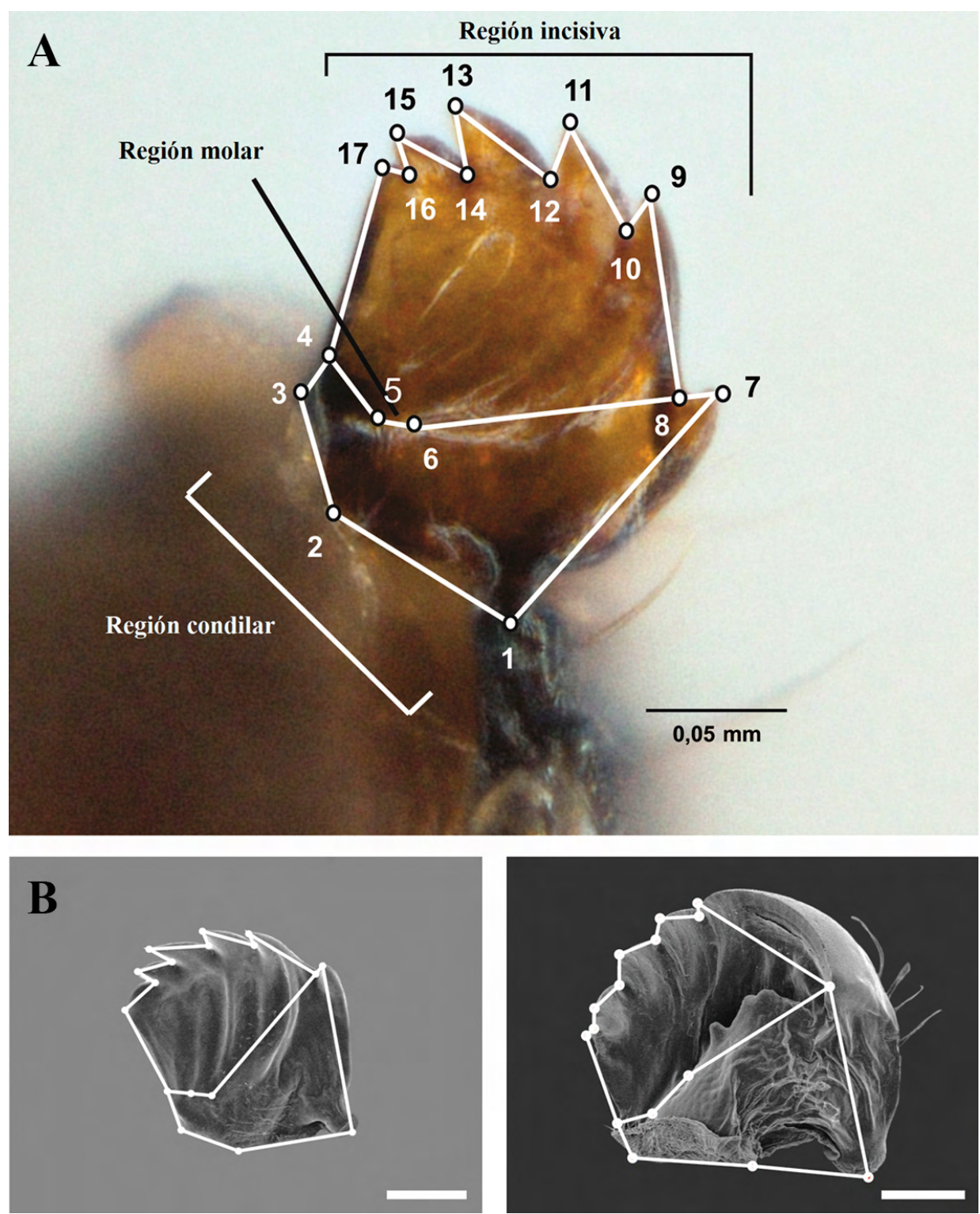

Figura 1. A. Mandíbula de primer instar de H. erato, vista mediana. Los números se refieren a las coordenadas usadas en los análisis morfométricos. B. Localización de coordenadas en mandíbulas de primer (izquierda) y quinto (derecha) instar de $H$. erato por microscopía electrónica. Barras horizontales en la parte inferior derecha de las imágenes corresponden a $0,05 \mathrm{~mm}$.

minación de cada instar fueron superiores al $90 \%$ (Tabla 2). Dicha mandíbula cortadora presentó algunas modificaciones en los cinco instares larvales (que serán discutidos posteriormente) y se destacaron cinco patrones: 1) 6 dientes conspícuos en la región incisiva, que se tornaron más alargados a medida que trancurrió el desarrollo; 2) región molar poco desarrollada, prácticamente lisa en el primer instar, seguido por regiones molares conspicuas con crestas y tubérculos fuertemente erguidos en el quinto instar, 3) el dislocamiento de los dientes incisivos en dirección al cóndilo anterior de la mandíbula, 4) la fusión de la cúspide del diente 7 con su base 8 (coordenadas 7 y 8) en el quinto instar, 5) el vértice de la margen distal, localizado entre los dos cóndilos (coordenada 2), se prolongó en dirección opuesta a la región incisiva (Fig. 2) (Tabla 3).

Las larvas de quinto instar de $H$. erato alimentadas exclusivamente con $P$. actinia tuvieron cabezas más grandes que
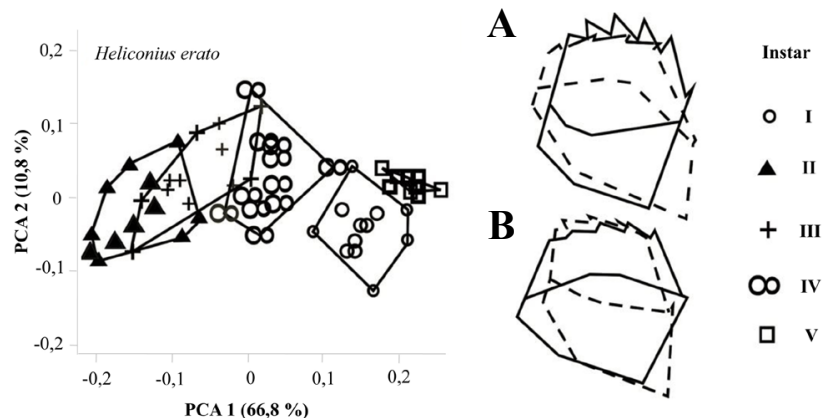

Figura 2. Análisis de componentes principales (PCA) de las formas mandibulares de los cinco instares larvales de $H$. erato. A. y B. representan las deformaciones por "Thin Plate Splines" del componente principal 1 (PC1) y componente principal 2 (PC2), respectivamente. La línea punteada representa o extremo negativo y la línea continua o extremo positivo de los componentes. 
aquellas alimentadas con las otras tres especies de Passiflora. Por otro lado, el tamaño de las mandíbulas y áreas de músculos aductores mandibulares de los individuos en todos los tratamientos no presentaron diferencias estadísticas significativas (Tabla 4) (Fig. 3).

El análisis de variables canónicas estructuró en media las diferentes plantas hospederas $(\mathrm{F}=2,183$, Wilks' $\lambda=0,259$, $\mathrm{P}=0,001$ ) (Fig. 4). Comparaciones MANOVA pareadas entre la forma mandibular de los individuos alimentados con las cuatro plantas mostró que los que comieron $P$. suberosa tienen mandíbulas significativamente diferentes de los que comieron $P$. actinia y $P$. misera. Las otras comparaciones no difirieron estadísticamente (Tabla 5).
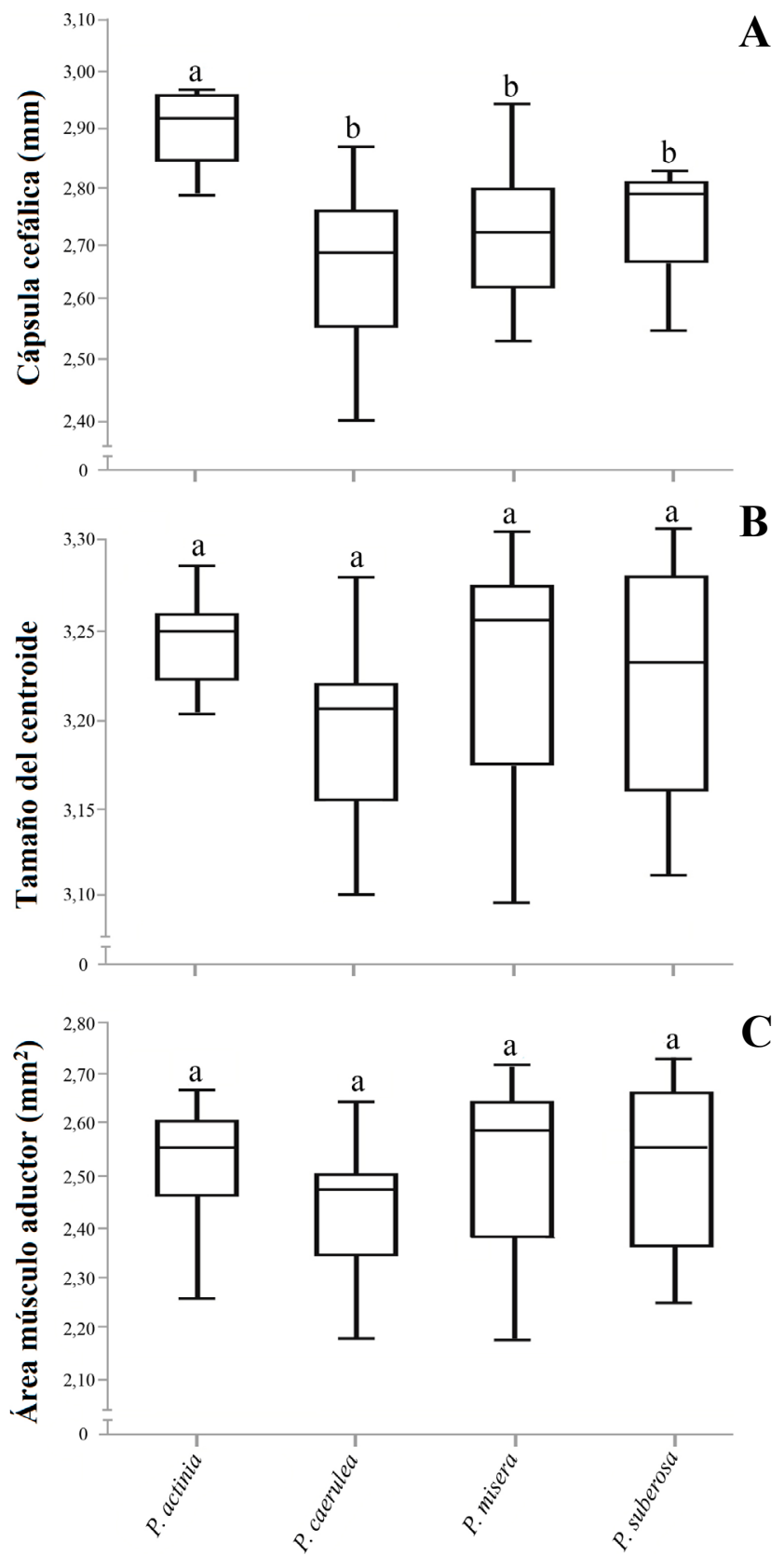

Especie de Passiflora

Figura 3. Longitud de: A. Cápsula cefálica. B. Tamaño de la mandíbula (tamaño del centroide). C. Área del músculo aductor mandibular de larvas de quinto instar de $H$. erato alimentadas con cuatro especies de Passiflora. Cajas con letras diferentes difieren estadísticamente (Kruskal-Wallis, seguido las pruebas de Dunn, $\mathrm{P}<0,05$ ).
Tabla 2. Porcentajes de identificación correcta de los cinco instares larvales de $H$. erato según el análisis discriminante (CVA).

\begin{tabular}{lccccc}
\hline \multirow{2}{*}{ Especie } & \multicolumn{5}{c}{ Clasificación correcta/instar larval (\%) } \\
\cline { 2 - 6 } & $\mathbf{L 1}$ & $\mathbf{L 2}$ & $\mathbf{L 3}$ & $\mathbf{L 4}$ & $\mathbf{L 5}$ \\
\hline H. erato & 100 & 90 & 100 & 100 & 100 \\
\hline
\end{tabular}

El análisis discriminante mostró porcentajes de identificación correcta superiores al $70 \%$ para cada una de las plantas hospederas $(71,43 \%$ para mandíbulas de larvas criadas en $P$. actinia, $71 \%$ en $P$. caerulea, $75 \%$ en $P$. misera y 78,68\% para larvas criadas en $P$. suberosa). El árbol (no enraizado) de distancias fenotípicas de Mahalanobis obtenido del análisis discriminante (CVA) ilustra dicha comparación al mostrar que la cría en $P$. misera y $P$. actinia originó larvas con mandíbulas muy similares entre sí y diferentes de las de los individuos criados en $P$. suberosa, los cuales divergen drásticamente del resto del grupo. Además, las mandíbulas de larvas alimentadas con $P$. caerulea aparecen como un grupo de "forma mandibular intermediaria" entre el grupo formado por los individuos de $P$. misera $+P$. actinia y el de los individuos de $P$. suberosa (Fig. 5).

Las rectas correspondientes al tamaño de la mandíbula y del músculo aductor asociado de larvas de quinto instar de $H$. erato en las cuatro plantas hospederas mostraron coeficientes alométricos negativos (Fig. 6) y se presentaron diferencias estadísticamente significativas entre las inclinaciones de las rectas correspondientes al área del músculo aductor y el tamaño de la mandíbula de los individuos (ANCOVAs, $\mathrm{P}<$ 0,0001) (Tabla 6).

\section{Discusión}

Las mandíbulas de larvas de $H$. erato fueron de tipo masticador (Alexander 1961) durante toda su ontogénesis, con algunas diferencias entre los cinco instares larvales. Teniendo en cuenta que las mandíbulas analizadas fueron extraídas de larvas sacrificadas casi que inmediatamente después de la muda, sin haberse alimentado, demuestra que dichas diferencias se atribuyen a su trayectoria ontogenética y no a desgastes ocasionados por el consumo de tejido vegetal.

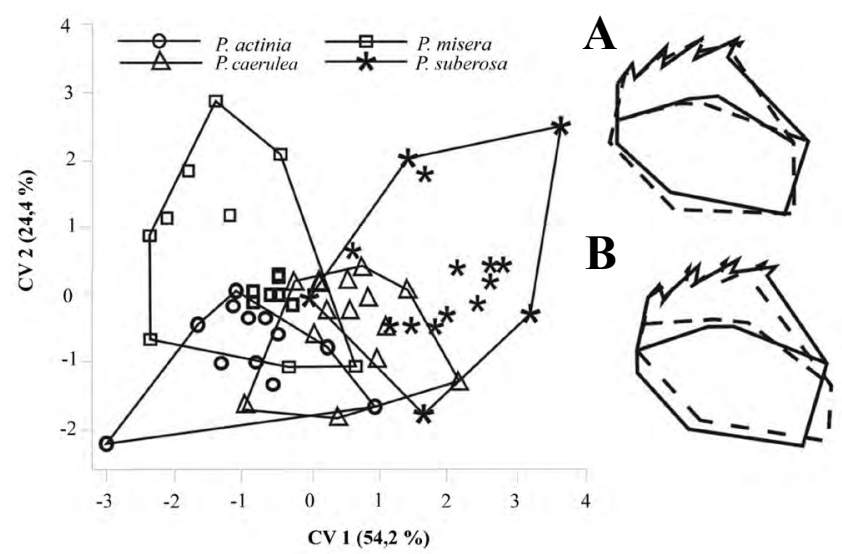

Figura 4. Análisis de variables canónicas (CVA) de la forma mandibular de larvas de quinto instar de Heliconius erato criadas en cuatro especies de Passiflora. CV1 y CV2 representan las variables canónicas 1 (A) y 2 (B). La línea punteada representa el extremo negativo y la continua el positivo de los componentes. 


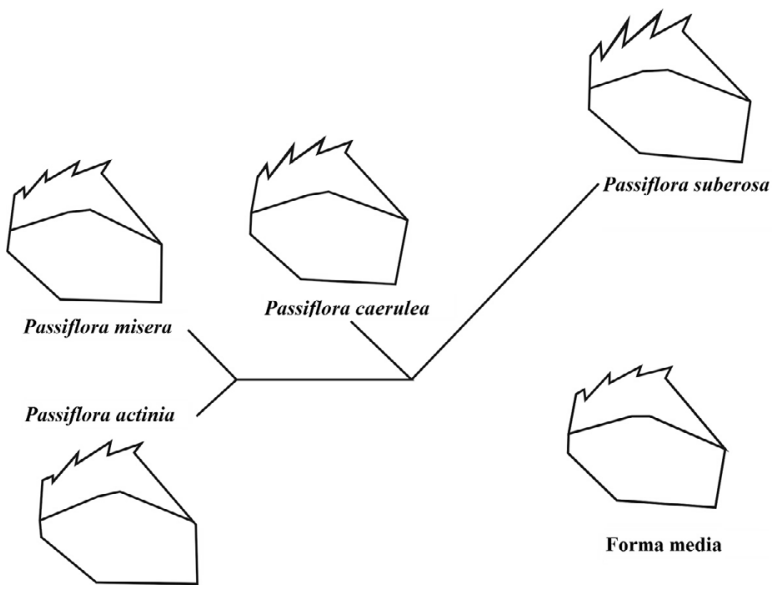

Figura 5. Arboles "neighbor-joining" (no enraizadas) de las distancias fenotípicas de Mahalanobis para la forma mandibular de larvas de quinto instar de $H$. erato alimentadas con cuatro especies de Passiflora. Las ilustraciones mandibulares asociadas a cada planta hospedera corresponden a la forma media obtenida por sobreposición de Procrustes (GPA) de las coordenadas mandibulares de individuos criados en cada hospedero vegetal y en el extremo inferior derecho la forma media total de los datos, sin discriminación de la planta hospedera usada como alimento por los individuos.

La hembra de $H$. erato pone huevos en regiones blandas de la planta hospedera, como zarcillos y hojas jóvenes (Périco y Araújo 1991) garantizando que las larvas recién eclosionadas se alimenten con éxito pues, como fue demostrado por Rodrigues y Moreira (1999), gran parte de larvas pequeñas obligadas a comer hojas maduras (que son naturalmente más rígidas) mueren durante los primeros días de vida. En ese sentido, los estudios morfológicos de Silveira (2002) revelan que las mandíbulas de larvas de $H$. erato al inicio del desarrollo son fuertemente dentadas, sin estructuras accesorias y, por tanto, incapaces de triturar alimentos rígidos, lo que explicaría la mortalidad cuando son sometidas a alimentación con hojas duras. A medida que transcurre la ontogénesis larval, los individuos se alimentan progresivamente de hojas cada vez más duras (Rodrigues y Moreira 1999) se sugiere que el desarrollo de crestas y tubérculos conspicuos en la región molar de las mandíbulas de larvas de quinto instar, observados en este estudio, posiblemente es lo que les permite que trituren y consuman hojas rígidas.

Diversos autores sugieren que las adaptaciones mandibulares en insectos herbívoros están sujetas a sus hábitos alimenticios y a las características del recurso utilizado por los mismos (Snodgrass 1935; Bernays 1991) ya que las mandíbulas son las únicas estructuras encargadas de seleccionar y cortar el alimento en fragmentos menores que puedan ser ingeridos (Chapman 1998). Según esto, hojas con diferentes niveles de dureza requerirían diferentes niveles de esfuerzo por parte del herbívoro (Elpino-Campos 2010); hojas más duras necesitarian de más esfuerzo y una musculatura mandibular mayor y, en contraste, hojas más suaves no demandarían un incremento de la musculatura mandibular (Bernays 1986).

Considerando solamente las hospederas viables para la especie, $P$. misera tiene hojas más suaves y menos nutritivas que $P$. suberosa (Kerpel 2004); pese a esto, P. misera es la planta que confiere mejor desarrollo larval a $H$. erato (Menna-Barreto y Araújo 1985; Périco 1995; Kerpel 2004). En lo que se refiere a dureza de las hojas, las pasifloráceas utilizadas en este estudio varian significativamente (Silveira

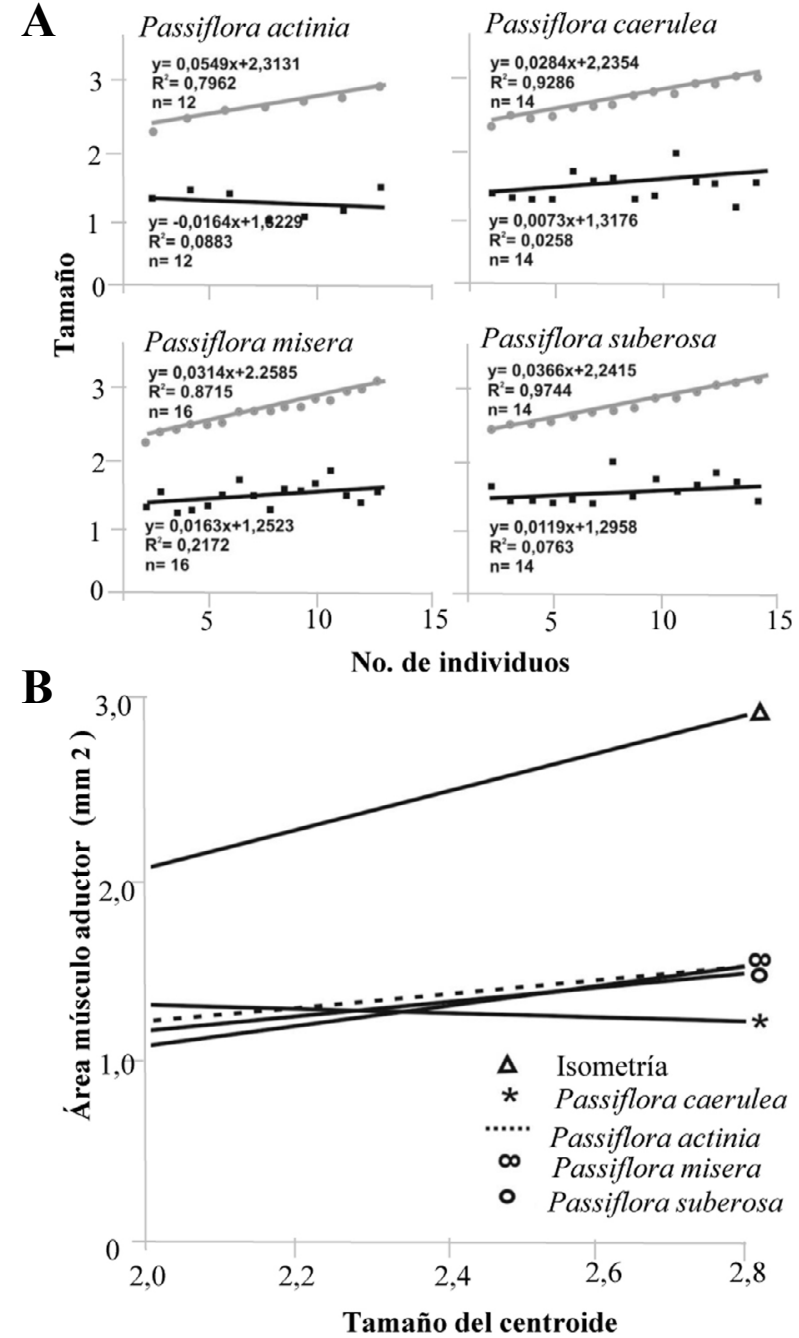

Figura 6. A. Rectas de crecimiento mandibular (gris) y crecimiento del área del músculo aductor de la mandíbula (negro) de larvas de quinto instar de $H$. erato criadas en cuatro especies de Passiflora. B. Variación del área del músculo aductor mandibular en relación al tamaño mandibular (tamaño del centroide) de los individuos. Comparaciones entre las rectas correspondientes al área del músculo y la recta del tamaño mandibular en cada hospedero, usando el total de datos, difieren significativamente en su inclinación (ANCOVAs, $\mathrm{P}<0,0001$ ).

2002; Kerpel 2004; Elpino-Campos 2010). Nuestros resultados muestran que las larvas de $H$. erato alimentadas desde el inicio de su desarrollo en dichas plantas no sufrieron cambios significativos en las dimensiones de sus mandíbulas ni en los músculos aductores. Además, independiente de las plantas hospederas, los músculos aductores crecieron proporcionalmente menos que las mandíbulas en todas las larvas, lo que puede deberse a que la energía metabólica invertida en cambios morfológicos sea totalmente direccionada a originar modificaciones en la forma mandibular, especificamente, y no necesariamente en su tamaño ni en el tamaño de los músculos (Molen et al. 2007). En este estudio, los cambios de forma propiamente dicha (ver definición usada en morfometría geométrica en Molen et al. 2007) evidenciados son discutidos a continuación.

Pese a que el efecto de la planta hospedera no repercutió en el tamaño de la mandíbula, si lo hizo en su forma, la cual fue diferente dependiendo de la Passiflora que las larvas comieron. Como fue registrado en la literatura, se esperaría que 
Tabla 3. Descripción morfológica de las mandíbulas de primer (L1) y quinto (L5) instar de H. erato.

\begin{tabular}{|c|c|c|c|}
\hline \multirow{2}{*}{ Especie } & \multirow{2}{*}{$\begin{array}{c}\text { Nivel de } \\
\text { agregación }\end{array}$} & \multicolumn{2}{|c|}{ Instar larval } \\
\hline & & L1 & $\mathbf{L 5}$ \\
\hline \multirow[t]{5}{*}{ Heliconius erato } & Solitario & $\begin{array}{l}\text { Seis dientes conspicuos en el área incisiva. Región molar } \\
\text { poco desarrollada (casi plana). }\end{array}$ & $\begin{array}{l}\text { Mandíbula aplanada. } \\
\text { Seis dientes conspicuos en la región incisiva. }\end{array}$ \\
\hline & & & $\begin{array}{l}\text { Formación de superficie aplanada entre los dientes } \\
7 \text { y } 9 \text { de la región incisiva. }\end{array}$ \\
\hline & & Tipo de corte de las mandíbulas: CORTADOR & $\begin{array}{l}\text { Región molar fuertemente elevada, presentando } \\
\text { crestas y tubérculos largos al parecer fusionados y } \\
\text { erguidos en la superficie, en dirección a la región } \\
\text { incisiva. }\end{array}$ \\
\hline & & Tejido consumido: Hojas jóvenes y zarcillos. & $\begin{array}{l}\text { Tipo de corte de las mandíbulas: CORTADOR } \\
\text { TRITURADOR }\end{array}$ \\
\hline & & & Tejido consumido: Hojas jóvenes y hojas maduras. \\
\hline
\end{tabular}

las larvas alimentadas con $P$. suberosa, con hojas más duras (Kerpel 2004), tuvieran dientes incisivos más lisos cuando se comparan con los individuos criados en los otras tres hospederas, que poseen hojas más suaves y menos densas (ElpinoCampos 2010). Esto no sucedió. Pese a que mandíbulas con dientes aplanados (o ausentes) se asocian a insectos que se alimentan de hojas duras (Bernays y Janzen1988), nuestros resultados revelaron que los individuos que comieron $P$. suberosa, de hojas más duras, desarrollaron dientes más largos y conspicuos que los criados en $P$. caerulea y $P$. misera, que tienen hojas más suaves.
Otro aspecto importante, es que los individuos de $H$. erato consumen progresivamente hojas más duras a medida que el desarrollo larval avanza (Elpino-Campos 2010) por lo que cambios en la resistencia y dureza de las mandíbulas a medida que la ontogénesis transcurre también serían esperados, mas fueron descartadas por Elpino-Campos en estudio detallado realizado en 2010. La plasticidad fenotípica entendida como una capacidad adaptativa de los individuos a las condiciones que les son impuestas, siempre a partir de un fenotipo preexistente (Whtiman y Ananthakrishnan 2009) nos lleva a sugerir que los dientes más pronunciados en las lar-

Tabla 4. Análisis de variación con los valores para las pruebas de Kruskal-Wallis y de Dunn correspondientes a las comparaciones entre las cabezas de larvas de quinto instar de $H$. erato criadas con cuatro especies de Passiflora. El asterisco indica diferencias estadísticas significativas.

\begin{tabular}{|c|c|c|c|c|}
\hline \multirow{2}{*}{ Planta hospedera } & \multicolumn{4}{|c|}{ p - valor prueba de Dunn } \\
\hline & P. actinia & P. caerulea & P. misera & P. suberosa \\
\hline \multicolumn{5}{|l|}{ Tamaño cabeza (mm) } \\
\hline \multicolumn{5}{|l|}{ Kruskal-Wallis $=14,59$} \\
\hline \multicolumn{5}{|l|}{$\mathrm{P}=0,002201^{*}$} \\
\hline P. actinia & - & $0,00116^{*}$ & $0,002336^{*}$ & $0,001941 *$ \\
\hline P. caerulea & & - & 0,466 & 0,2406 \\
\hline P. misera & & & - & 0,3176 \\
\hline P. suberosa & & & & - \\
\hline \multicolumn{5}{|c|}{ Tamaño mandíbula (centroide) } \\
\hline \multicolumn{5}{|l|}{ Kruskal-Wallis $=3,592$} \\
\hline \multicolumn{5}{|l|}{$P=0,309$} \\
\hline P. actinia & - & 0,1171 & 0,8151 & 0,9702 \\
\hline P. caerulea & & - & 0,1239 & 0,168 \\
\hline P. misera & & & - & 0,9007 \\
\hline P. suberosa & & & & - \\
\hline \multicolumn{5}{|l|}{ Área músculo adutor (mm2) } \\
\hline \multicolumn{5}{|l|}{ Kruskal-Wallis $=2,180$} \\
\hline \multicolumn{5}{|l|}{$\mathrm{p}=0,5359$} \\
\hline P. actinia & - & 0,3132 & 0,1326 & 0,2626 \\
\hline P. caerulea & & - & 0,7867 & 0,8720 \\
\hline P. misera & & & - & 0,9336 \\
\hline P. suberosa & & & & - \\
\hline
\end{tabular}


Tabla 5. Comparaciones MANOVA pareadas entre las mandíbulas de larvas de quinto instar de $H$. erato alimentadas con cuatro pasifloráceas. El asterisco indica diferencias estadísticas significativas.

\begin{tabular}{|c|c|c|c|c|}
\hline $\begin{array}{c}\text { Especie de Passiflora } \\
\text { consumida }\end{array}$ & Wilks` $\lambda$ & $\mathbf{F}$ & p - valor & $\begin{array}{l}\text { p - Corrección } \\
\text { de Bonferroni }\end{array}$ \\
\hline P. actinia $\mathrm{x}$ P. caerulea & 0,5476 & 0,8261 & 0,6158 & 0,6158 \\
\hline P. actinia $\times$ P. misera & 0,4526 & 1,4517 & 0,2670 & 0,5340 \\
\hline P. actinia $\mathrm{x}$ P. suberosa & 0,1358 & 6,3666 & $0,00363 *$ & $0,0180 *$ \\
\hline P. caerulea $\mathrm{x}$ P. misera & 0,4964 & 1,9279 & 0,1047 & 0,3141 \\
\hline P. caerulea $\mathrm{x}$ P. suberosa & 0,4314 & 2,2404 & 0,0688 & 0,0688 \\
\hline P. misera $\mathrm{x}$ P. suberosa & 0,3034 & 4,3619 & $0,0028^{*}$ & $0,0171^{*}$ \\
\hline
\end{tabular}

vas alimentadas con $P$. suberosa son una optimización de la mandíbula cortadora, preexistente en estos individuos, para maximizar el efecto de "corte" de las hojas rígidas. En otros hospederos como $P$. misera y $P$. caerulea, los individuos presentan dientes menos conspicuos; pues al ser hospederos de hojas más blandas (Elpino-Campos 2010) el acceso estaría facilitado y las adaptaciones plásticas no serían requeridas en la mandíbula.

Passiflora suberosa, P. misera y P. caerulea ya fueron usadas en estudios de desarrollo larval de $H$. erato (Rodrigues y Moreira 1999; Elpino-Campos 2010; Kerpel 2004; Mugrabi-Oliveira y Moreira 1996b; Périco y Araújo 1991; Périco 1995; Menna-Barreto y Araújo 1985) mostrando que, en algunas regiones donde las tres plantas están disponíbles, $P$. misera es el hospedero en el cual las larvas llegaron más rapidamente al estado adulto (Menna-Barreto y Araújo 1985; Périco 1995; Rodrigues y Moreira 2002, 2004) a pesar de tener una menor cantidad de nutrientes que las otras hospederas (e.g. P. suberosa) (Kerpel 2004). Por tal motivo, las larvas necesitan consumir mucho más tejido para compensar el déficit nutricional y, en consecuencia, gastan más tiempo alimentándose (Elpino-Campos 2012). En términos energéticos, consumir hojas blandas es más favorable, aunque la calidad del hospedero sea menor, pues es más barato alimentarse de tejido suave por más tiempo, que invertir metabolicamente en adaptaciones mandibulares que permitan ingerir hojas más duras y más nutritivas, como fue el caso de los individuos alimentados exclusiva y obligatoriamente con $P$. suberosa.

Predicciones sobre la evolución del aparato bucal de larvas de $H$. erato con relación al consumo de $P$. actinia no fueron posibles debido a la inexistencia de datos experimentales previos sobre la dureza de sus hojas. Sin embargo, nuestros resultados muestran que individuos alimentados con hojas de

Tabla 6. Comparaciones alométricas (ANCOVAs) de las inclinaciones $(\beta)$ e interceptos $(\alpha)$ correspondientes a las rectas de crecimiento del músculo aductor mandibular vs. tamaño mandibular (centroide) de larvas de $H$. erato alimentadas con cuatro especies de Passiflora. Asterisco indica diferencias estadísticas significativas.

\begin{tabular}{lcccc}
\hline \multirow{2}{*}{$\begin{array}{c}\text { Especie } \\
\text { Passiflora }\end{array}$} & \multicolumn{1}{c}{$\boldsymbol{\beta}$} & \multicolumn{2}{c}{$\boldsymbol{\alpha}$} \\
\cline { 2 - 5 } & F & P & F & P \\
\hline P. actinia & 46,6283 & $<0,0001^{*}$ & - & - \\
P. caerulea & 12,1794 & $0,0009037^{*}$ & - & - \\
P. misera & 12,8187 & $0,0006676^{*}$ & - & - \\
P. suberosa & 16,9249 & $0,0001184^{*}$ & - & - \\
\hline
\end{tabular}

esta planta poseen mandíbulas similares a los alimentados con $P$. misera, sugiriendo que $P$. actinia sería una planta con características similares, de hojas suaves y blandas, lo que aún necesita ser corroborado en futuros estudios.

\section{Conclusiones}

Se demostró que la planta hospedera utilizada como alimento durante la ontogénesis de larvas de la mariposa $H$. erato puede moldear la forma de sus mandíbulas como respuesta a una adaptación plástica de los individuos para acceder al recurso vegetal de una forma más eficiente. En este estudio, larvas alimentadas exclusivamente con las hojas de $P$. suberosa, que son más duras, desarrollaron dientes significativamente más largos que los individuos alimentados con las otras pasifloráceas, consideradas más suaves. Lo anterior contradice lo propuesto por otros autores de que dientes largos y prolongados no son aptos para consumir tejido vegetal rígido y son propios de especies herbívoras que comen hojas blandas. Sugerimos que el aumento en la longitud de los dientes inducido por comer $P$. suberosa junto con el desarrollo de crestas y tubérculos en la región molar de las mandíbulas que $H$. erato, más que una contradicción a la literatura conocida, puede entenderse como una adecuación que parte de una condición morfológica preexistente (mandíbula cortadora), mediado por plasticidad fenotípica, que es más barata en términos energéticos y optimiza el proceso de alimentación de estos insectos.

\section{Literatura citada}

ADAMS, D. C.; ROSENBERG, M. S. 1998. Partial warps, phylogeny, and ontogeny: a comment on Fink and Zelditch (1995). Systematic Biology, Washington 47 (1): 168-173.

ALEXANDER, A. J. 1961. A study of the biology and behavior of the caterpillars, pupae and emerging butterflies of the subfamily Heliconiinae in Trinidad, West Indies. Part I. Some Aspects of larval behavior. Zoologica 46 (1): 1-24.

AYMONE, A. C. 2006. Alometria cefálica na ontogênese de Heliconius erato phyllis (Fabricius) (Lepidoptera: Nymphalidae: Heliconiinae). Dissertação de bacharelado apresentada ao programa de Pós-Graduação em Biologia Animal. Universidade Federal do Rio Grande do Sul. 37 p.

AYMONE, A. C. 2009. Restrições ontogenéticas na evolução da forma da cápsula cefálica em oito espécies de heliconíneos (Lepidoptera: Nymphalidae). Dissertação de mestrado apresentada ao programa de Pós-Graduação em Biologia Animal. Universidade Federal do Rio Grande do Sul. 107 p.

BAINES, R. A.; TYRER, N. M.; DOWNER, R. G. H. 1990. Serotoninergic innervation of the locust mandibular closer muscle 
modulates contractions though the elevation of cyclic adenosine monophosphate. Journal of Comparative Neurology 294 (4): 623-632.

BARP, E. A.; SOARES, G. L. G.; GOSMANN, G.; MACHADO, A. M.; VECCHI, C.; MOREIRA, G. R. P. 2006. Phenotypic plasticity in Passiflora suberosa L. (Passifloraceae): Induction and reversion of two morphs by variation in light intensity. Brazilian Journal of Biology 66 (3): 853-862.

BAYLAC, M. 2008. Rmorph: a R geometric and multivariate morphometrics library. [Disponible por el autor en: baylac@mnhn.fr].

BENSON, W. W.; BROWN, K. S. JR.; GILBERT, L. E. 1976. Coevolution of plants and herbivores: passion flowers butterflies. Evolution 29 (1): 659-680.

BENSON, W. W. 1978. Resource partitioning in passion on vine butterflies. Evolution 32 (1): 493-518.

BERNAYS, E. A. 1986. Diet-induced head allometry among foliage-chewing insects and its importance for graminivores. Science 231 (4737): 495-497.

BERNAYS, E. A.; JANZEN, D. H. 1988. Saturniid and sphingid caterpillars: two ways to eat leaves. Ecology 69 (4): 1153-1160.

BERNAYS, E. A. 1991. Evolution of insect morphology in relation to plants. Philosophical Transactions of the Royal Society of London Series B- Biological Sciences 333 (1): 257-264.

BERNAYS, E. A. 1998. Evolution of feeding behavior in insect herbivores. BioScience 48 (1): 35-44.

BRADSHAW, A. D. 1965. Evolutionary significance of phenotypic plasticity in plants. Advances in Genetics 13 (1): 115-155.

CHAPMAN, R. F. 1995. Mechanics of food handling by chewing insects. pp. 3-31. In: Chapman, R. F.; Boer, G. (Eds.). Regulatory mechanisms in insect feeding. New York, Chapman \& Hall, $398 \mathrm{p}$.

CHAPMAN, R. F. 1998. The insects: structure and function. Harvard University Press, Cambridge. 919 p.

DEVELOPMENT CORE TEAM. 2008. R: A language and Environment for Statistical Computing. R Foundation for Statistical Computing, Vienna. http://www.R-project.org. [Fecha revisión: Julio 2017].

DEWITT, T. J.; SCHEINER, S. M. 2004. Phenotypic plasticity: Functional and conceptual approaches. Oxford University Press, New York. 247 p.

ELPINO-CAMPOS, A. 2010. Características da planta hospedeira, ontogênese e comportamento alimentar de larvas de Heliconius erato phyllis (Fabricius, 1975) (Lepidoptera: Nymphalidae). Dissertação de doutorado apresentada ao programa de PósGraduação em Biologia Animal. Universidade Federal do Rio Grande do Sul. 122 p.

ELPINO-CAMPOS, A. 2012. Feeding behavior of Heliconius erato phyllis (Fabricius) (Lepidoptera: Nymphalidae) larvae on passion vines. Acta Ethologica 15 (1): 1-12.

FENNY, P. 1970. Seasonal changes in oak leaf tannins and nutrients as a cause of spring feeding by winter moth caterpillars. Ecology 51 (4): $565-581$.

GILBERT, L. E. 1975. Ecological consequences of a coevolved mutualism between butterflies and plants. pp. 210-240. In: Gilbert, L. E.; Raven, P. H. (Eds.). Coevolution of animals and plants. University of Texas Press, Austin.

GOTTHARD, K.; NYLIN, S. 1995. Adaptive plasticity and plasticity as an adaptation: A selective review of plasticity in animal morphology and life history. Oikos 74 (1): 3-17.

GRISS, C. 1990. Mandibular motor neurons of the caterpillar of the hawk moth Manduca sexta. Journal of Comparative Neurology 296 (3): 393-402.

JORGE, L. R.; CORDEIRO-ESTRELA, P.; KLACZKO, L. B.; MOREIRA, G. R. P.; FREITAS A. V. L. 2011. Host-plant dependent wing phenotypic variation in the neotropical butterfly Helicnius erato. Biological Journal of the Linnean Society 102 (4): 765-774.

KERPEL, S. M. 2004. Influencia do conteúdo nutricional de Passiflora suberosa e Passiflora misera na performance, nutrição, di- gestão e comportamento de escolha de Heliconius erato phyllis (Lepidoptera: Nymphalidae). Tese de doutorado apresentada ao programa de Pós-Graduação em Ecologia. Universidade Federal do Rio Grande do Sul. 163 p.

LARSSON, S. 2002. Resistance in trees to insects - an overview of mechanisms and interactions. pp. 1-29. In: Wagner, M. R.; Clancy, K. M.; Lieutier, F.; Paine, T. D. (Eds.). Mechanisms and deployment of resistance in trees to insects. Springer, Dordrecht.

MAHALANOBIS, P. C. 1936. On the Generalized Distance in Statistics. Journal of Genetics 41: 159-193.

MASUKO, K. 1986. Motor innervations and proprioceptors on the mouthparts in the worker honeybee Apis mellifera: I. Mandibular nerve. Journal of Morphology 188 (1): 51-68.

MENNA-BARRETO, Y.; ARAÚJO, A. M. 1985. Evidence for host plant preferences in Heliconius erato phyllis from southern Brazil (Nymphalidae). Journal of Research on the Lepidoptera, Arcadia 24 (1): 41-46.

MOLEN, S. V. D.; ABADIAS, N. M.; GONZÁLES-JOSÉ, R. 2007. Introducción a la morfometría geométrica. Curso teórico práctico. 1 edición. Puerto Madryn y Barcelona: Editorial FCNyMUNLP. 78 p.

MUGRABI-OLIVEIRA, E.; MOREIRA, G. R. P. 1996a. Conspecific mimics and low hostplant availability reduce egg laying by Heliconius erato phyllis (Lepidoptera: Nymphalidae). Revista Brasileira de Zoologia 13 (1): 929-937.

MUGRABI-OLIVEIRA, E.; MOREIRA, G. R. P. 1996b. Size of and damage on shoots of Passiflora suberosa (Passifloraceae) influence oviposition side selection of Heliconius erato phyllis (Lepidoptera: Nymphalidae). Revista Brasileira de Zoologia 13 (1): 939-953.

PAUL, J.; GRONENBERG, W. 2002. Motor control of the mandible closer muscle in ants. Journal of Insect Physiology 48 (2): 255-267.

PÉRICO, E.; ARAÚJO, A. M. 1991. Suitability of host plants (Passifloraceae) and their acceptableness by Heliconius erato and Dryas iulia (Lepidoptera: Nymphalidae). Evolución Biológica 5 (1): 59-74.

PÉRICO, E. 1995. Interação entre quatro especies de Heliconiini (Lepidoptera: Nymphalidae) e suas plantas hospedeiras (Passifloraceae) em uma floresta secundária do Rio Grande do Sul, Brasil. Biociências, Porto Alegre 3 (1): 3-18.

PIGLIUCCI, M. 2002. Phenotypic plasticity: beyond nature and nurture. Jonhs Hopkins University Press, Baltimore 328 p.

PRICE, P. W. 1997. Insect ecology, New York. John Wileyand Sons. $874 \mathrm{p}$.

REHDER, V. 1989. Sensory pathways and motoneurons of the proboscis reflex in the subesophageal ganglion of the honeybee. Journal of Comparative Neurology 279 (3): 499-513.

RODRIGUES, D.; MOREIRA, G. R. P. 1999. Feeding preference of Heliconius erato phyllis (Lepidoptera, Nymphalidae) in relation to leaf and consequences for larval performance. Journal of the Lepidopterists' Society 53 (3): 108-113.

RODRIGUES, D.; MOREIRA, G. R. P. 2002. Geographical variation in larval host-plant use by Heliconius erato (Lepidoptera, Nymphalidae) and consequences for adult life history. Brazilian Journal of Biology 62 (2): 321-322.

RODRIGUES, D.; MOREIRA, G. R. P. 2004. Seasonal variation in larval host-plants and consequences for Heliconius erato (Lepidoptera: Nymphalidae) adult body size. Austral Ecology 29 (4): 437-445.

ROHLF, F. J. 2006. TpsPLS, Version 1.18. Stony Brook, Department of Ecology and Evolution, State University of New York. Disponible en: http://life.bio.sunysb.edu/morph/ [Fecha revisión: julio 2017].

ROHLF, F. J. 2010. TpsDig. Stony Brook, Department of Ecology and Evolution, State University of New York.

ROHLF, F. J.; SLICE, D. 1990. Extensions of the Procrustcs method for the optimal superimposition of landmarks. Systematic Zoology 39 (1): 40-59. 
SACCO, J. C. 1962. Flora ilustrada do Rio Grande do Sul - Passifloraceae. Boletin ICN 12 (1): 7-29.

SACCO, J. C. 1980. Passifloráceas. Flora ilustrada Catarinense 1, 3-130 p.

SAITOU, N.; NEI, M. 1987. The neighbor-joining method: A new method for reconstructing phylogenetic trees. Molecular Biology and Evolution 4 (4): 406-425.

SCHILICHTING, C. D. 1986. The evolution of phenotipic plasticity in plants. Annual Review of Ecology Evolution and Systematics 17 (1): 667-693.

SCHEINER, S. M. 1993. Genetics and evolution of phenotypic plasticity. Annual Review of Ecology and Systematics 24 (1): 35-68.

SILVEIRA, M. A. P. A. 2002. Variação da dureza da folha em Passiflora, efeito no desgaste das mandíbulas de Heliconius erato phyllis (Lepidoptera: Nymphalidae) e consequências sobre a herbivoria. Porto Alegre, Dissertação de Mestrado em Biologia Animal. Universidade Federal do Rio Grande do Sul, 44 p.

SNODGRASS, R. E. 1935. Principles of insect morphology. McGraw Hill Company, New York. 667 p.

SNODGRASS, R. E. 1950. Comparative studies on the jaws of mandibulate arthropods. Smithsonian Miscellaneous Collection 116 (1): 1-85.

SULTAN, E. 1995. Phenotypic plasticity and plant adaptation. Acta Botanica Neerlandica 44 (4): 363-383.
VEGLIANTE, F. 2005. Larval head anatomy of Heterogynis penella (Zygaenoidea, Heterogynidae), and a general discussion of caterpillar head structure (Insecta, Lepidoptera). Acta Zoologica 86 (3): 167-194.

WEST-EBERHARD, M. J. 2003. Development plasticity and evolution. Oxford University press, New York. 816 p.

WHITMAN, W. W.; ANANTHAKRISHNAN, T. N. 2009. Phenotypic plasticity of insects, mechanisms and consequences. Science Publishers, United States of America 1. 894 p.

WRIGHT, S. 1931. Evolution in Mendelian populations. Genetics 16 (2): $97-159$.

Recibido: 01-oct-2017 • Aceptado: 24-may-2018

Citación sugerida:

MILLÁN, C.; FORNEL, R.; MOREIRA, G. R. P. 2018. Plasticidad fenotípica en mandíbulas de Heliconius erato (Lepidoptera: Nymphalidae) inducida por diferentes plantas hospederas (Passifloraceae). Revista Colombiana de Entomología 44 (2): 273 282. Julio-Diciembre 2018. 